\title{
Intra-tumoral genomic heterogeneity in rectal cancer: mutational status is dependent on preoperative biopsy depth and location.
}

Floris A. Vuijk ( $\sim$ f.a.vuijk@lumc.nl )

Leids Universitair Medisch Centrum https://orcid.org/0000-0001-7888-924X

Carlijn van de Water

Radboudumc

Shannon van Lent - van Vliet

Radboudumc

Maxime J.M. van der Valk

Leids Universitair Medisch Centrum

Cornelis J.H. van de Velde

Leids Universitair Medisch Centrum

Alexander L. Vahrmeijer

Leids Universitair Medisch Centrum

Iris D. Nagtegaal

Radboudumc

Denise E. Hilling ( $\triangle$ D.E.Hilling@lumc.nl)

Leids Universitair Medisch Centrum

\section{Research article}

Keywords: rectal cancer, intra-tumor heterogeneity, next generation sequencing

Posted Date: April 9th, 2020

DOI: https://doi.org/10.21203/rs.3.rs-20696/v1

License: (c) (1) This work is licensed under a Creative Commons Attribution 4.0 International License. Read Full License

Version of Record: A version of this preprint was published at Cancers on May 9th, 2021. See the published version at https://doi.org/10.3390/cancers13092271. 


\section{Abstract}

Background Neoadjuvant therapy is indicated for all patients with locally advanced rectal cancer, even though a significant number of patients show minimal or no response. Adequate response prediction before the start of neoadjuvant treatment might reduce unnecessary waiting periods and therapy related toxicity in non-responders. Genomic mutational status might provide a means to predict response to neoadjuvant therapy. However, it is unclear whether predictions based on genomic mutational status in single preoperative biopsies are reliable due to intra-tumoral heterogeneity. In this study we aim to investigate the reliability of genomic mutations found in single pre-operative biopsies by comparing these genomic mutations to 4 other locations within the same tumor using next generation sequencing. Methods Rectal cancer patients undergoing primary resection, without neoadjuvant therapy, were included. Of all patients, one biopsy, two deep and two superficial samples were obtained and sequenced using a targeted next generation sequencing gene panel. Concordance between these 5 samples was assessed. Results $\mathbb{\text { In }}$ this feasibility study we included 11 patients. In 7 out of 11 (64\%) patients, all 5 samples showed concordant mutations. In 4 out of 11 patients (36\%) disconcordant mutations were observed. Conclusions Evaluation of genomic mutational status on a single pre-operative biopsy is inadequate in a substantial amount of the patients and is therefore insufficient to predict response to neoadjuvant therapy. Predictive algorithms including multiple parameters might be required to further stratify patients to optimal treatment regimens.

\section{Background}

Locally advanced rectal cancer (LARC) patients are currently treated with neoadjuvant (chemo)radiotherapy followed by surgical resection (1). In clinical practice, the observed response to neoadjuvant therapy is heterogeneous. A pathological complete response (complete regression of tumor and/or pathological lymph nodes) is seen in $15-20 \%$ (up to $73 \%$ in some series) of patients, whereas in the vast majority of patients (54-75\%) neoadjuvant therapy results in a partial response $(2,3)$.

Unfortunately, a subset of $10-50 \%$ of LARC patients receives futile neoadjuvant treatment when minimal or no response is observed $(2,4)$. Currently, treatment is stratification as advised in national guidelines is based on clinical TNM stage, tumor distance to the mesorectal fascia and the presence of extramural vascular invasion (5). Response prediction based on parameters readily available before neoadjuvant treatment might provide a means to ensure patient-tailored treatment, and reduce unnecessary waiting periods and therapy related toxicity in non-responders.

Tumor associated immune response and intra-tumoral heterogeneity might be involved in causing therapeutic resistance of the tumor (6). Intra-tumoral genomic heterogeneity refers to the presence of genetically distinct sub clones within cancer lesions, and is developed by tumors in reaction to a diversity of microenvironmental factors including hypoxia, tissue stiffness, immune response and chronic inflammation or can be caused by the polyclonal origin of these tumors $(7,8)$. Intra-tumoral genomic heterogeneity is particularly significant in colorectal cancer, and is attributed to the presence of both microsatellite- and chromosomal instability (9-11). 
In previous studies the predictive value of (a combination of) clinical, pathological and radiological parameters in predicting response to (neoadjuvant) therapy has been assessed. Among these are several pathological expression indices (e.g. KI-67, EGFR and P21) and genomic mutational status, as well as radiological parameters derived from MR and [ ${ }^{18}$ F]FDG-PET/CT imaging (e.g. standardized uptake value (SUV) and apparent diffusion coefficient (ADC)(12-20). Unfortunately, these studies so far have not resulted in clinically used prediction models.

The predictive value of genomic mutations in colorectal cancer has previously been investigated, concluding that KRAS, as well as RAS, BRAF and PIK3CA mutations, are predictive of tumor response to anti-EGFR therapy $(17,18,21-25)$. Furthermore, a high degree of intra-tumoral genomic heterogeneity (as investigated by whole exome sequencing) has been associated with worse disease-free survival and was correlated with a higher rate of liver metastases (26). So far, no specific genomic mutations have been found to accurately predict response to neoadjuvant therapy in LARC patients (19).

A combination of genomic mutations might provide a means to predict response to neoadjuvant therapy in (locally advanced) rectal cancer patients before the start of neoadjuvant therapy. However, the reliability of next generation sequencing performed on routinely obtained single preoperative biopsies has yet to be established. Intra-tumoral heterogeneity has been shown to be significant in rectal tumors and their associated lymph nodes and metastases $(27,28)$. Therefore, genomic mutations found in single preoperative biopsies might vary within individual patients, depending on the biopsy location and depth. It is therefore currently unclear whether predictions based on single preoperative biopsies are reliable to predict response to neoadjuvant therapy.

In this study we aim to investigate the reliability of genomic mutations found in a single preoperative biopsy by comparing these mutations to 4 other locations within the same tumor using next generation sequencing.

\section{Methods}

\section{Patients}

Rectal cancer patients from the Radboud University Medical Center, Nijmegen, the Netherlands and diagnosed between 2010 and 2012 with a biopsy confirmed rectal adenocarcinoma were retrospectively included in this study. To prevent any influence of neoadjuvant therapy on the results, patients undergoing surgical resection of the primary tumor without neoadjuvant chemo- and/or radiotherapy were included.

Patient characteristics were obtained from medical records, including age, gender, clinical- and pathological characteristics. This project was conducted in accordance with the Declaration of Helsinki, and did not require approval of the local IRB according to local WMO regulations.

\section{Tumor identification and DNA isolation}


For each patient, five tissue samples were obtained from representative formalin-fixed paraffin-embedded (FFPE) tumor blocks containing material of 1 preoperative diagnostic biopsy, 2 superficial tumor tissue samples and 2 deep (central) tumor tissues samples of the resected specimen. Optimal FFPE blocks (with adequate tumor cellularity of $\geq 20 \%$ from full samples, and $>10 \%$ in biopsy samples) for smMIP analysis were identified and marked by an expert pathologist (I.N.) on representative hematoxylin and eosin (H\&E) stained slides. To obtain sufficient genomic DNA, marked tumor areas were cut out from 10 sequential (non-stained) slides (each $6 \mu \mathrm{m}$ thick). DNA was isolated at $56^{\circ} \mathrm{C}$ for 1 hour using TET-lysis buffer with 5\% Chelex-100 (Bio-Rad, Hercules, USA) and $400 \mu \mathrm{g}$ proteinase K (Qiagen, Valencia, USA), followed by inactivation at $95^{\circ} \mathrm{C}$ during 10 minutes (29). The DNA concentration was determined using the Qubit High Sensitivity Kit (Invitrogen, Carlsbad, USA) per manufacturer's protocol.

\section{smMIP sequencing}

A panel of 911 smMIPs was used to detect variants in 31 cancer-related genes, as displayed in Table 1. To provide gender control, smMIPs targeting $A M E L X$ and $A M E L Y$ were included. The smMIP sequencing protocol has previously been clinically validated and used in the Radboud University Medical Center (29). One hundred nanogram of isolated DNA was included per sample. After sample preparation, manual library preparation was performed (29). The purified libraries were diluted. Sequencing was performed using the NextSeq500 (Illumina, San Diego, USA) per manufacturer's protocol (300 cycles High Output sequencing Kit, lllumina, San Diego, USA), resulting in $2 \times 150$ bp paired end reads.

\section{Sequence data analysis}

Sequence data was generated from the NextSeq500, after which Bcl to FASTQ conversion and demultiplexing of barcoded reads was automatically performed. Sequence Pilot software (JSI Medical Systems $\mathrm{GmbH}$, Ettenheim, Germany) was used for generating consensus reads and variant identification, with settings as previously described (29). Variants found in samples passing gender control and exceeding an average minimum reading depth of 180 were automatically filtered with an inhouse Python script, as depicted in Fig. 1. This threshold excludes, with a certainty of $>95 \%$, the presence of a mutation at minimally $10 \%$ mutant allele frequency within covered regions.

\section{Statistical analysis}

Statistical analysis was performed using SPSS version 23 (SPSS, Inc., Chicago, USA). Numerical data is presented as mean (standard deviation) or median (interquartile range) based on distribution. Categorical data is presented as frequencies and percentages. In order to quantify tumor heterogeneity, differences in mutational status between biopsy, deep and superficial tumor samples were analyzed by calculating the percentages of concordance and disconcordance. Concordance was defined as all five samples ( 1 biopsy, 2 deep samples, and 2 superficial samples) showing identical (or no) mutations. Disconcordance was defined as $\geq 1$ mutation(s) in either of the 5 samples, which was not found in (one of) the other samples. For all tests performed, $\mathrm{P}<0.05$ was considered statistically significant.

\section{Results}




\section{Patients}

Data and tissue of 11 patients were included in this study. Patients were on average $72 \pm 27.4$ years old, and consisted of 6 men and 5 females. Of these, 9 had a pT3 tumor and 2 a pT4 tumor. All patients were treated with immediate resection of the rectal tumor, without prior chemo- and/or radiotherapy. The rectal tumor was on average located $57.8 \pm 46.3 \mathrm{~mm}$ from the anal verge, and measured $53.5 \pm 21.6 \mathrm{~mm}$ in diameter. Detailed clinicopathological features are summarized in Table 2.

\section{Mutation concordance}

Thirty-three genomic mutations were found in the following 8 genes: $A P C(9 / 11), B R A F(1 / 11), F B X W 7$ (2/11), KRAS (7/11), PIK3CA (1/11), PTEN (6/11), SMAD4 (1/11) and TP53 (6/11). Insufficient (partial) reading depth was found in biopsy samples of 3 patients (patient 5,8 and 9 ).

In 7 out of 11 (64\%) patients, all 5 samples showed concordant mutations. In 4 out of 11 patients (36\%) a disconcordance in mutations was observed within the 5 samples.

In patient 2 a disconcordance in KRAS, SMAD4 and TP53 mutations was found between the superficial sample and the biopsy as well as both deep samples. Patient 4 showed disconcordance as the TP53 mutation was only found in the biopsy and one of two superficial samples. Patient 5 showed disconcordance as the $A P C$ mutation was only found in the superficial samples compared to the deep samples (biopsy results were not available). In patient 8 disconcordance was found as different TP53 mutations were found in the biopsy compared to the deep and superficial samples. These results are depicted in Fig. 2.

\section{Discussion}

Response to neoadjuvant therapy is heterogeneous in LARC patients $(2,4)$. Currently, neoadjuvant therapy is indicated for all LARC patients, even though a significant subset of patients is therapy resistant. Adequate prediction of the response to neoadjuvant treatment might improve personalized treatment, and avoid unnecessary waiting periods and therapy related toxicity in non-responding patients. In addition, it might open up new opportunities to apply neoadjuvant therapy in a selected subset of well responding early stage tumors (without an indication for neoadjuvant therapy according to current guidelines). To ensure clinical translation of this response prediction, the use of readily available clinical, radiological and/or pathological parameters is desired. Genomic mutational status can be assessed on pre-operative biopsies which are routinely obtained in the diagnostic work up.

As this and previous research indicates, the use of a single predictive biomarker often underestimates the complex mechanisms involved in response prediction. In this study, genomic mutations in pre-operative biopsies were compared to 4 other locations within the same tumor using next generations sequencing. In $36 \%$ of the patients, evaluation of genomic mutational status on a single pre-operative biopsy has 
shown to be inadequate. This illustrates the genomic variability in rectal cancer and explains the difficulties in obtaining reliable biomarkers for response prediction. These results are in line with previous evidence supporting the presence of intra-tumoral genomic heterogeneity in a considerable proportion of rectal cancers (30). Three previous studies have compared genomic mutations in up to 3 intra tumoral locations. Hardiman et al. reported up to 10 coding variants uniquely corresponding to one of 3 of the tumor locations in their study of 6 patients (30). In the study of Bettoni et al., only $27 \%$ of the observed mutations corresponded to all three samples of a single rectal adenocarcinoma in one patient (31). On the other hand, Dijkstra et al. reported no differences in mutational status between deep and superficial colorectal cancer tissue in 30 patients (32).

This study has several limitations. First of all, the small sample size and limited targeted next generation sequencing panel might influence the interpretation of results. The number of disconcordant cases might actually be higher, as this targeted gene panel only provides information on a selected number of mutations. Furthermore, there is no $100 \%$ certainty the found mutations were not germ-line mutations, however considering the observed allelic frequency this is very unlikely. Furthermore, since the main aim of this study was to determine the reliability of response prediction based on a single preoperative biopsy rather than define the degree of intra-tumoral genomic heterogeneity, the influence on our results would be limited. To increase the reliability of the biopsy analysis, the use of multiple and possibly even deeper/larger preoperative biopsies might provide a better representation of intra-tumoral heterogeneity, but might also increase the risk of procedure related complications. A second possibility might be the application of whole exome sequencing or larger targeted gene panels (such as the TSO500, Illumina, San Diego, USA), as this possibly provides a more elaborate analysis of genomic mutations, as compared to next generation sequencing using a limited targeted gene panel. Using these techniques, the mutantallele heterogeneity (MATH) score was developed to quantitatively assess the spread of allele frequencies, and has been correlated to response $(19,33)$. However, as sampling errors are innate to the biopsy technique, parameters derived from full tumor imaging might be preferable to incorporate characteristics of all genetic sub clones present in these cancers.

Although the use of a single predictive parameter may be the most clinically usable method, it seems to underestimate the complexity of cancer biology. Previous research on the application of other predictive parameters (e.g. MRI, PET, radiomics feature analysis, metabolomics (34-37)) has up until now not resulted in the routine use of such predictive parameters, as these do not provide sufficient accuracy yet (13).

To overcome this challenge and address these complexities, data should probably be combined into a response predictive algorithm. The first steps towards identifying reliable parameters to be included in such models have already been made by Santos et al. in 2017, using multivariable analysis of both blood and tissue biomarkers (38). In such models, features derived from clinical, radiological, pathological and more advanced analysis such as radiomics and metabolomics can be incorporated. The downside of such studies is the need of large datasets acquired under a variety of conditions, as to be applicable in a myriad of clinical scenarios. 


\section{Conclusion}

In conclusion, assessment of overall tumor heterogeneity on a single pre-operative biopsy seems inadequate and the use of it to predict response to neoadjuvant therapy is warranted. As we aim for tailored treatment and optimized patient outcome, predictive algorithms including multiple features will have to be developed to further stratify patients to optimal treatment regimens.

\section{Abbreviations}

LARC Locally advanced rectal cancer

SUV Standardized uptake value

ADC Apparent diffusion coefficient

FFPE $\quad$ Formalin fixed paraffin embedded

H\&E Hematoxylin and eosin

MATH Mutant allele tumor heterogeneity

\section{Declarations}

Ethics approval: This study was conducted in accordance with the declaration of Helsinki, and did not require approval of the local IRB according to local WMO regulations.

Consent for publication: Not applicable.

Availability of data and materials: The datasets generate and analyzed in this study are not publicly available for the reason of protecting patients' privacy, but are available from the corresponding authors on reasonable request.

Competing interests: The authors of this manuscript have nothing to disclose.

Funding: This study was supported by the Bas Mulder Award (Granted to dr. D.E. Hilling, no. UL20157966). The funder (Dutch Cancer Society) was not further involved in this study, and only provided financial support.

Author contributions: FV, MV, IN and DH were in charge of the study design, data review and manuscript preparation. CW and SV contributed to data analysis and figure preparation. CV and AV contributed to the manuscript preparation. All authors read and approved the final manuscript.

Acknowledgements: Not applicable.

\section{References}


1. Beets-Tan RGH, Lambregts DMJ, Maas M, Bipat S, Barbaro B, Curvo-Semedo L, et al. Magnetic resonance imaging for clinical management of rectal cancer: Updated recommendations from the 2016 European Society of Gastrointestinal and Abdominal Radiology (ESGAR) consensus meeting. Eur Radiol. 2018 Apr;28(4):1465-75.

2. Maas M, Nelemans PJ, Valentini V, Das P, Rödel C, Kuo L-J, et al. Long-term outcome in patients with a pathological complete response after chemoradiation for rectal cancer: a pooled analysis of individual patient data. Lancet Oncol. 2010 Sep;11(9):835-44.

3. Appelt AL, Pløen J, Harling H, Jensen FS, Jensen LH, Jørgensen JCR, et al. High-dose chemoradiotherapy and watchful waiting for distal rectal cancer: a prospective observational study. Lancet Oncol. 2015 Aug;16(8):919-27.

4. Park J-S, Baek J-H, Lee W-S, Yang J-Y, Lee W-K, Kim K-K, et al. Long-term oncologic outcomes in pathologic tumor response after neoadjuvant chemoradiation for locally advanced rectal cancer. Korean J Clin Oncol. 2018 Jun;30(1):37-42. 14(.

5. Landelijke Werkgroep Gastro Intestinale Tumoren. Oncoline [Internet]. [cited 2019 Nov 6]. Available from: .

6. Frydrych LM, Ulintz P, Bankhead A, Sifuentes C, Greenson J, Maguire L, et al. Rectal cancer subclones respond differentially to neoadjuvant therapy. Neoplasia. 2019 Oct 1;21(10):1051-62.

7. Hinohara K, Polyak K. Intratumoral Heterogeneity: More Than Just Mutations. Trends Cell Biol. 2019 Jul 1;29(7):569-79.

8. Parsons BL. Many different tumor types have polyclonal tumor origin: evidence and implications. Mutat Res. 2008 Oct;659(3):232-47.

9. Molinari C, Marisi G, Passardi A, Matteucci L, De Maio G, Ulivi P. Heterogeneity in Colorectal Cancer: A Challenge for Personalized Medicine? Int J Mol Sci [Internet]. 2018 Nov 23 [cited 2019 Nov 6];19(12). Available from: .

10. Grady WM, Carethers JM. Genomic and epigenetic instability in colorectal cancer pathogenesis. Gastroenterology. 2008 Oct;135(4):1079-99.

11. Vogelstein B, Papadopoulos N, Velculescu VE, Zhou S, Diaz LA, Kinzler KW. Cancer genome landscapes. Science. 2013 Mar;29(6127):1546-58. 339(.

12. Habr-Gama A, São Julião GP, Gama-Rodrigues J, Vailati BB, Ortega C, Fernandez LM, et al. Baseline T Classification Predicts Early Tumor Regrowth After Nonoperative Management in Distal Rectal Cancer After Extended Neoadjuvant Chemoradiation and Initial Complete Clinical Response. Dis Colon Rectum. 2017 Jun;60(6):586-94.

13. Joye I, Deroose CM, Vandecaveye V, Haustermans K. The role of diffusion-weighted MRI and (18)FFDG PET/CT in the prediction of pathologic complete response after radiochemotherapy for rectal cancer: a systematic review. Radiother Oncol J Eur Soc Ther Radiol Oncol. 2014 Nov;113(2):158-65.

14. Giralt J, Eraso A, Armengol M, Rosselló J, Majó J, Ares C, et al. Epidermal growth factor receptor is a predictor of tumor response in locally advanced rectal cancer patients treated with preoperative radiotherapy. Int J Radiat Oncol. 2002 Dec 1;54(5):1460-5. 
15. Reerink O, Karrenbeld A, Plukker JTM, Verschueren RCJ, Sluiter MBGS. WJ, et al. Molecular Prognostic Factors in Locally Irresectable Rectal Cancer Treated Preoperatively by Chemoradiotherapy. Anticancer Res. 2004 Jan;24(2C)(3):1217-22.

16. Tsang JS, Vencken S, Sharaf O, Leen E, Kay EW, McNamara DA, et al. Global DNA methylation is altered by neoadjuvant chemoradiotherapy in rectal cancer and may predict response to treatment A pilot study. Eur J Surg Oncol EJSO. 2014 Nov;1(11):1459-66. 40(.

17. Lièvre A, Bachet J-B, Le Corre D, Boige V, Landi B, Emile J-F, et al. KRAS mutation status is predictive of response to cetuximab therapy in colorectal cancer. Cancer Res. 2006 Apr 15;66(8):3992-5.

18. Linardou H, Dahabreh IJ, Kanaloupiti D, Siannis F, Bafaloukos D, Kosmidis P, et al. Assessment of somatic k-RAS mutations as a mechanism associated with resistance to EGFR-targeted agents: a systematic review and meta-analysis of studies in advanced non-small-cell lung cancer and metastatic colorectal cancer. Lancet Oncol. 2008 Oct;9(10):962-72.

19. Greenbaum A, Martin DR, Bocklage T, Lee J-H, Ness SA, Rajput A. Tumor Heterogeneity as a Predictor of Response to Neoadjuvant Chemotherapy in Locally Advanced Rectal Cancer. Clin Colorectal Cancer. 2019 Jun;18(2):102-9.

20. Oh BY, Shin H-T, Yun JW, Kim K-T, Kim J, Bae JS, et al. Intratumor heterogeneity inferred from targeted deep sequencing as a prognostic indicator. Sci Rep. 2019 Mar;14(1):1-8. 9(.

21. De Roock W, Claes B, Bernasconi D, De Schutter J, Biesmans B, Fountzilas G, et al. Effects of KRAS, BRAF, NRAS, and PIK3CA mutations on the efficacy of cetuximab plus chemotherapy in chemotherapy-refractory metastatic colorectal cancer: a retrospective consortium analysis. Lancet Oncol. 2010 Aug;11(8):753-62.

22. Jhawer M, Goel S, Wilson AJ, Montagna C, Ling Y-H, Byun D-S, et al. PIK3CA mutation/PTEN expression status predicts response of colon cancer cells to the epidermal growth factor receptor inhibitor cetuximab. Cancer Res. 2008 Mar 15;68(6):1953-61.

23. Tian S, Simon I, Moreno V, Roepman P, Tabernero J, Snel M, et al. A combined oncogenic pathway signature of BRAF, KRAS and PI3KCA mutation improves colorectal cancer classification and cetuximab treatment prediction. Gut. 2013 Apr;62(4):540-9.

24. Sartore-Bianchi A, Martini M, Molinari F, Veronese S, Nichelatti M, Artale S, et al. PIK3CA mutations in colorectal cancer are associated with clinical resistance to EGFR-targeted monoclonal antibodies. Cancer Res. 2009 Mar 1;69(5):1851-7.

25. Douillard J-Y, Oliner KS, Siena S, Tabernero J, Burkes R, Barugel M, et al. Panitumumab-FOLFOX4 treatment and RAS mutations in colorectal cancer. N Engl J Med. 2013 Sep 12;369(11):1023-34.

26. Joung J-G, Oh BY, Hong HK, Al-Khalidi H, Al-Alem F, Lee H-O, et al. Tumor Heterogeneity Predicts Metastatic Potential in Colorectal Cancer. Clin Cancer Res Off J Am Assoc Cancer Res. 2017 Dec 1;23(23):7209-16.

27. Baldus SE, Schaefer K-L, Engers R, Hartleb D, Stoecklein NH, Gabbert HE. Prevalence and heterogeneity of KRAS, BRAF, and PIK3CA mutations in primary colorectal adenocarcinomas and 
their corresponding metastases. Clin Cancer Res Off J Am Assoc Cancer Res. 2010 Feb 1;16(3):7909.

28. Losi L, Baisse B, Bouzourene H, Benhattar J. Evolution of intratumoral genetic heterogeneity during colorectal cancer progression. Carcinogenesis. 2005 May;26(5):916-22.

29. Eijkelenboom A, Kamping EJ, Kastner-van Raaij AW, Hendriks-Cornelissen SJ, Neveling K, Kuiper RP, et al. Reliable Next-Generation Sequencing of Formalin-Fixed, Paraffin-Embedded Tissue Using Single Molecule Tags. J Mol Diagn. 2016 Nov 1;18(6):851-63.

30. Hardiman KM, Ulintz PJ, Kuick RD, Hovelson DH, Gates CM, Bhasi A, et al. Intra-tumor genetic heterogeneity in rectal cancer. Lab Invest. 2016 Jan;96(1):4-15.

31. Bettoni F, Masotti C, Habr-Gama A, Correa BR, Gama-Rodrigues J, Vianna MR, et al. Intratumoral Genetic Heterogeneity in Rectal Cancer: Are Single Biopsies representative of the entirety of the tumor? Ann Surg. 2017;265(1):e4-6.

32. Dijkstra JR, Tops BBJ, Nagtegaal ID, van Krieken JHJM, Ligtenberg MJL. The homogeneous mutation status of a 22 gene panel justifies the use of serial sections of colorectal cancer tissue for external quality assessment. Virchows Arch Int J Pathol. 2015 Sep;467(3):273-8.

33. Mroz EA, Rocco JW. MATH, a novel measure of intratumor genetic heterogeneity, is high in pooroutcome classes of head and neck squamous cell carcinoma. Oral Oncol. 2013 Mar;49(3):211-5.

34. Zhou X, Yi Y, Liu Z, Cao W, Lai B, Sun K, et al. Radiomics-Based Pretherapeutic Prediction of Nonresponse to Neoadjuvant Therapy in Locally Advanced Rectal Cancer. Ann Surg Oncol. 2019;26(6):1676-84.

35. Yi X, Pei Q, Zhang Y, Zhu H, Wang Z, Chen C, et al. MRI-Based Radiomics Predicts Tumor Response to Neoadjuvant Chemoradiotherapy in Locally Advanced Rectal Cancer. Front Oncol [Internet]. 2019 Jun 26 [cited 2019 Nov 6];9. Available from: .

36. Giannini V, Mazzetti S, Bertotto I, Chiarenza C, Cauda S, Delmastro E, et al. Predicting locally advanced rectal cancer response to neoadjuvant therapy with 18F-FDG PET and MRI radiomics features. Eur J Nucl Med Mol Imaging. 2019 Apr;46(4):878-88.

37. Jia H, Shen X, Guan Y, Xu M, Tu J, Mo M, et al. Predicting the pathological response to neoadjuvant chemoradiation using untargeted metabolomics in locally advanced rectal cancer. Radiother Oncol $\mathrm{J}$ Eur Soc Ther Radiol Oncol. 2018;128(3):548-56.

38. Santos MD, Silva C, Rocha A, Nogueira C, Castro-Poças F, Araujo A, et al. Predictive clinical model of tumor response after chemoradiation in rectal cancer. Oncotarget. 2017 Jul 28;8(35):58133-51.

\section{Tables}

Table 1. Overview of Regions Targeted by the Transcan smMIP Panel. 


\begin{tabular}{|c|c|c|c|c|c|}
\hline Gene & $\begin{array}{l}\text { Transcript ID } \\
\text { (RefSeq) }\end{array}$ & $\begin{array}{l}\text { Transcript ID } \\
\text { (Ensembl) }\end{array}$ & $\begin{array}{l}\text { Exon } \\
\text { number }\end{array}$ & Targeted regions & $\begin{array}{l}\text { Positions analyzed } \\
\text { for variants }\end{array}$ \\
\hline \multirow[t]{2}{*}{$A C V R 1 B$} & ENST00000257963 & NM_004302 & 02 & Activin types I and II receptor domain & c. $92-5$ to c. $331+5$ \\
\hline & & & 03-09 & Transforming growth factor beta type I GS-motif & c. 556 to c. $1518+5$ \\
\hline$A C V R 2 A$ & ENST00000241416 & NM_001616 & $06-11$ & Protein kinase domain & c. $673-5$ to c. $1542+5$ \\
\hline AMER 1 & ENST00000330258 & NM_152424 & 02 & WTX Protein & c. 639 to c. 1629 \\
\hline$A P C$ & ENST00000257430 & NM_000038 & $01-16$ & Whole gene & c $1-5$ to c. $8532+5$ \\
\hline \multirow[t]{2}{*}{ ARID1A } & ENST00000324856 & NM_006015 & $11-12$ & ARID DNA-binding domain & c.2989 to c.3397 \\
\hline & & & 20 & SWI/SNF-like complex subunit BAF250/Osa & c.5820 to c.6777 \\
\hline$B 2 M$ & ENST00000558401 & NM_004048 & 02 & Immunoglobulin C1-set domain & c. $68-5$ to c. $346+5$ \\
\hline$B R A F$ & ENST00000288602 & NM_004333 & 15 & Codon D594-K601 & c. $1742-5$ to $1860+5$ \\
\hline CASP5 & ENST00000393141 & NM_004347 & $02-03$ & CARD domain & c. 8 to $433+5$ \\
\hline CASP8 & ENST00000358485 & NM_001080125 & 07-09 & Caspase domain & c. 838 to $1617+5$ \\
\hline \multirow[t]{2}{*}{ CTNNB1 } & ENST00000349496 & NM_001904 & 03 & Codon D32-S45 & c.36 to c.163 \\
\hline & & & 08 & Codon W383-N387 & c. $1082-5$ to c. $1185+5$ \\
\hline \multirow[t]{2}{*}{$E G F R$} & ENST00000275493 & NM_005228 & 12 & Receptor L domain & c. 1391 to c. $1498+5$ \\
\hline & & & $18-21$ & Protein tyrosine kinase & c. $2062-5$ to $2625+5$ \\
\hline ERBB2 & ENST00000269571 & NM_004448 & $18-24$ & Protein tyrosine kinase & c. 2101 to c. $2970+5$ \\
\hline$F B X W 7$ & ENST00000281708 & NM_033632 & $07-12$ & WD domain, G-beta repeat & c. 1035 to c. $2124+5$ \\
\hline GNAS & ENST00000371085 & NM_000516 & $08-09$ & Codon R201 and Q227 & c. $586-5$ to c. $718+5$ \\
\hline IDH2 & ENST00000330062 & NM_002168 & 04 & Codon R140 and R172 & c. $374-5$ to c. $534+5$ \\
\hline \multirow[t]{3}{*}{ KRAS } & ENST00000311936 & NM_004985 & 02 & Codon G12, and G13 & c. $1-5$ to $\mathrm{c} .111+5$ \\
\hline & & & 03 & Codon A59 and Q61 & c. $112-5$ to c. 232 \\
\hline & & & 04 & Codon K117 and A146 & $\begin{array}{l}\text { c. } 291-5 \text { to c. } 385 \text { and } \\
\text { c. } 402 \text { to c. } 450+5\end{array}$ \\
\hline$M E T$ & ENST00000318493 & NM_001127500 & $15-21$ & Protein tyrosine kinase & c. 3140 to c $.4227+5$ \\
\hline \multirow[t]{2}{*}{$N R A S$} & ENST00000369535 & NM_002524 & 02 & Codon G12 and G13 & c. $1-5$ to c.99 \\
\hline & & & 03 & Codon A59 and Q61 & c. 135 to c. 272 \\
\hline \multirow[t]{2}{*}{ PIKЗCA } & ENST00000263967 & NM_006218 & 10 & Codon E542 to Q546 & c. 1557 to c. $1664+5$ \\
\hline & & & 21 & Codon M1043 to G1049 & c. 3041 to c. $3207+5$ \\
\hline POLE & ENST00000320574 & NM_006231 & $03-13$ & $\begin{array}{l}\text { DNA-directed DNA polymerase, family B, exonuclease } \\
\text { domain }\end{array}$ & c. $205-5$ to c. 1301 \\
\hline PTEN & ENST00000371953 & NM_000314 & $05-08$ & $\begin{array}{l}\text { Dual specificity phosphatase, catalytic domain, C2 } \\
\text { domain of PTEN tumor-suppressor protein }\end{array}$ & c. 310 to c. $1026+5$ \\
\hline$R N F 43$ & ENST00000407977 & NM_017763 & $02-10$ & Whole CDS & c. $1-5$ to c. $2352+5$ \\
\hline$S M A D 2$ & ENST00000262160 & NM_005901 & $02-11$ & Whole CDS & c. $1-5$ to c. $1404+5$ \\
\hline \multirow[t]{2}{*}{$S M A D 4$} & ENST00000342988 & NM_005359 & 03-04 & MH1 domain & c. $250-5$ to c. $454+5$ \\
\hline & & & $09-12$ & MH2 domain & c. $956-5$ to c. $1659+5$ \\
\hline \multirow[t]{2}{*}{$S M A R C A 2$} & ENST00000349721 & NM_003070 & $15-21$ & SNF2-related, N-terminal domain & c. $2185-5$ to $3078+5$ \\
\hline & & & $23-25$ & Helicase, C-terminal & c. 3136 to c. $3684+5$ \\
\hline \multirow[t]{2}{*}{$S M A R C A 4$} & ENST00000450717 & NM_001128846 & $15-21$ & SNF2-related, N-terminal domain & c. $2275-5$ to c. $3168+5$ \\
\hline & & & $23-25$ & Helicase, C-terminal & c. 3324 to c. $3374+5$ \\
\hline$S M A R C B 1$ & ENST00000263121 & NM_003073 & 05-09 & SNF5/SMARCB1/INI1 & c. $501-5$ to c. $1158+5$ \\
\hline SOX9 & ENST00000245479 & NM_000346 & 01-03 & Whole CDS & c. $1-5$ to c. $1530+5$ \\
\hline \multirow[t]{2}{*}{ TCF7L 2} & ENST00000369397 & NM_030756 & 01-06 & CTNNB1 binding, N-terminal & c. $1-5$ to c. $719+5$ \\
\hline & & & $09-10$ & High mobility group box domain & c. $933-5$ to c. $1200+5$ \\
\hline TGFBR2 & ENST00000359013 & NM_001024847 & 04 & Codon E125 & c. $339-5$ to c. $529+5$ \\
\hline TP53 & ENST00000269305 & NM_000546 & 03-08 & P53 DNA-binding domain & c. 83 to c. $919+5$ \\
\hline
\end{tabular}

Table 2. Patient characteristics. 


\begin{tabular}{lll}
\hline Variables & & N=11 \\
\hline Age (years) & Mean (SD) & $72.2(27.4)$ \\
Gender & Male & $6(55 \%)$ \\
pT & Female & $5(45 \%)$ \\
& 3 & $9(82 \%)$ \\
pN & 4 & $2(18 \%)$ \\
& 0 & $6(55 \%)$ \\
EMVI & 1 & $3(27 \%)$ \\
& 2 & $2(18 \%)$ \\
Differentiation & Yes & $4(36 \%)$ \\
& No & $6(55 \%)$ \\
Distance to CRM (mm) & Missing & $1(9 \%)$ \\
Diameter tumor (mm) & Good & $9(82 \%)$ \\
Total number of lymph nodes & Bad & $1(9 \%)$ \\
Number of tumor positive lymph nodes & Median (IQR) & $0(0-3)$ \\
Distance from anal verge (mm) & Mean (SD) & $57.8(46.3)$ \\
\hline
\end{tabular}

Abbreviations: SD, standard deviation; pT, clinical tumor stage; $\mathrm{pN}$, clinical nodal stage; EMVI, extramural vascular invasion; CRM, circumferential resection margin; IQR, interquartile range.

\section{Figures}




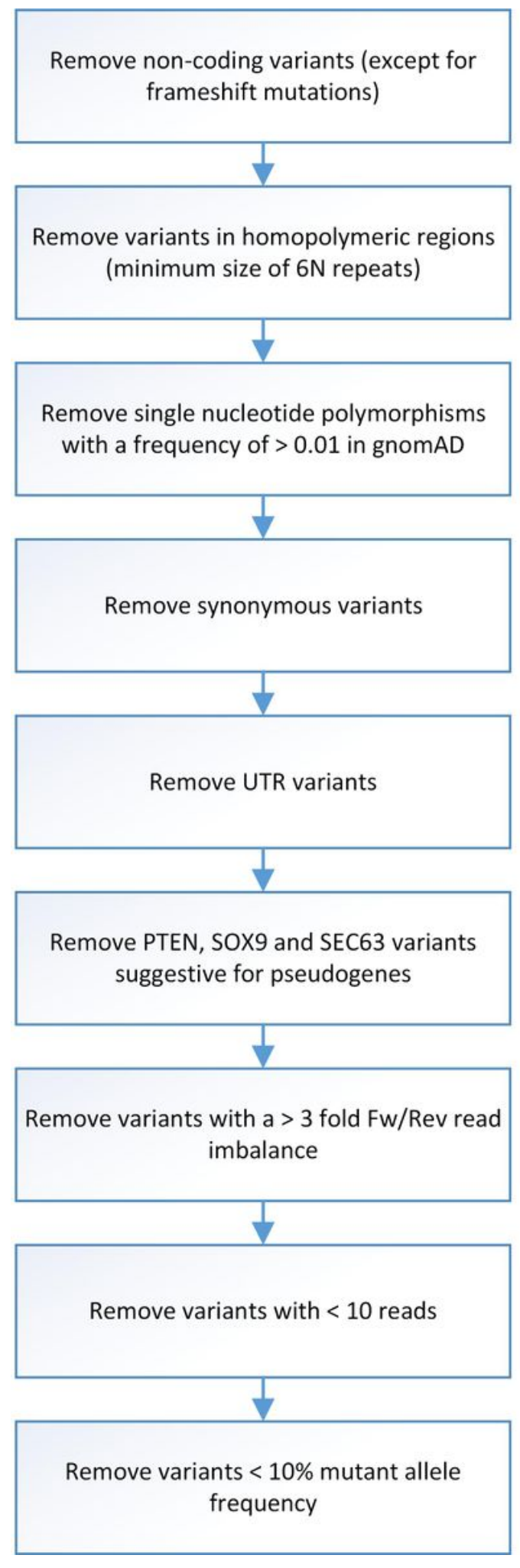

\section{Figure 1}

Flowchart of smMIP analysis data filtering. Overview of data filtering before smMIP data analysis. 


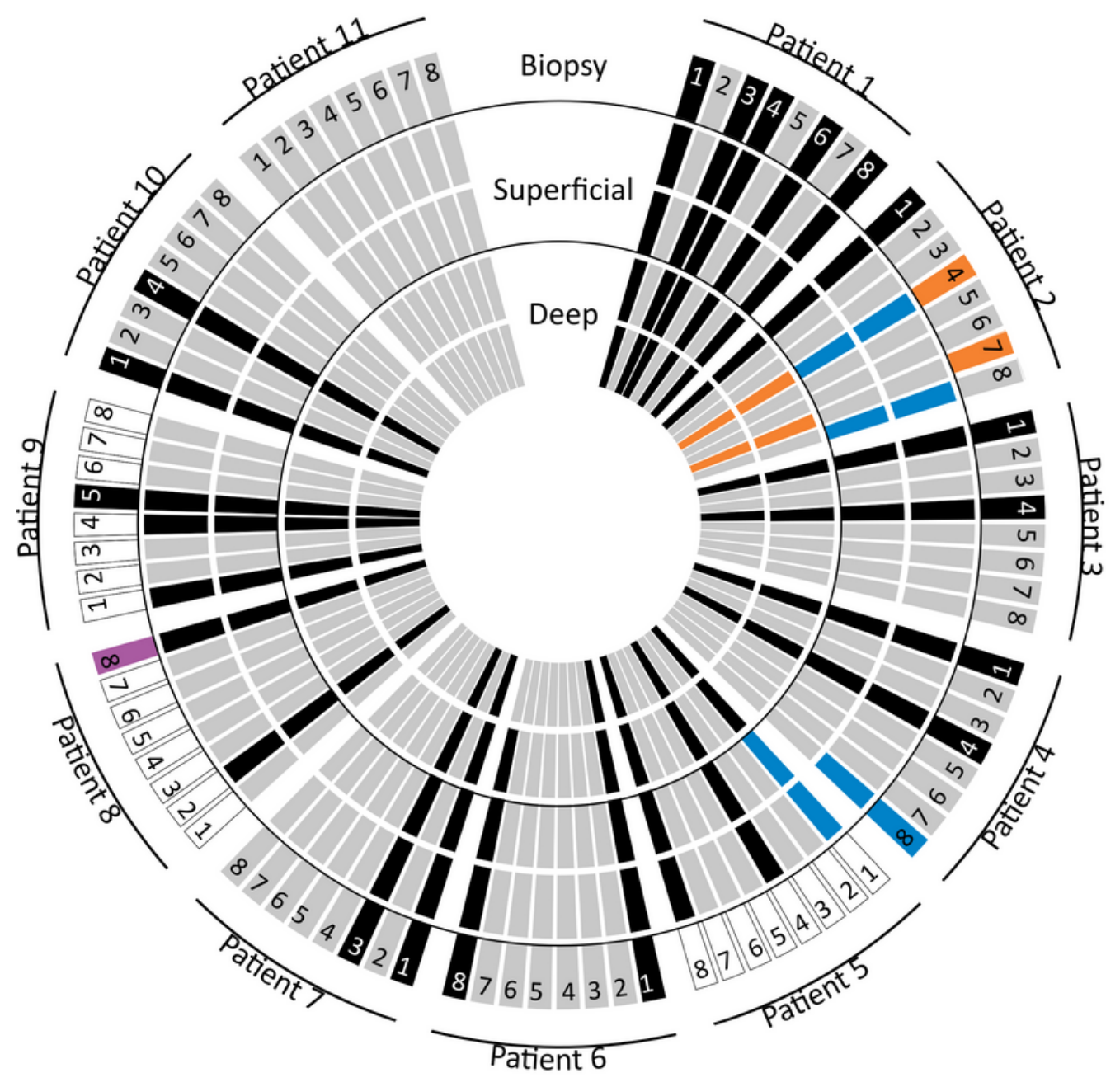

Shared alteration

Shared alteration

Wildtype

Insufficient read depth

Alteration exclusive to deeper samples

Alteration exclusive to superficial samples

Alteration exclusive to biopsy

1. $A P C$

2. $B R A F$

3. FBXW7

4. KRAS

5. PIK3CA

6. PTEN

7. SMAD4

8. $T P 53$

Figure 2

Graphical display of mutations in all samples. Representation of mutations found in deep, superficial and biopsy samples. 